\title{
Medication adherence in successful kidney transplant recipients
}

\author{
Todd M. Ruppar, MSN(R), RN, GCNS-BC and Cynthia L. Russell, RN, PhD \\ University of Missouri, Columbia, MO
}

\section{Abstract}

Objective-To explore the medication-taking behavior of successful kidney transplant recipients and determine what behaviors were common among this group.

\begin{abstract}
Methods-Open-ended interviews were conducted by telephone with 19 individuals who had successfully maintained a transplanted kidney for 25 years or more. Data were coded by using a grounded theory approach to explore patterns of phenomena, common descriptions, and relationships.
\end{abstract}

\begin{abstract}
Results-Four themes emerged as participants described the behaviors they developed to adhere successfully to the immunosuppressive medication required for maintaining their transplanted kidneys. The themes were reminder methods, obtaining medications, maintaining routines, and problem-solving strategies. Kidney transplant recipients identified the importance of developing and maintaining medication-taking skills and routines on medication adherence. Problem-solving abilities were also valuable during times of disruption of normal routines.
\end{abstract}

Conclusions-Interventions focusing on medication-taking skills, habit formation, and resources for problem solving may improve immunosuppressive medication adherence and clinical outcomes in kidney transplant recipients.

The World Health Organization defines medication adherence as "the extent to which a person's behaviour (taking medications, following a recommended diet and/or executing life-style changes) corresponds with the agreed recommendations of a health care provider."1 Adherence to an effective immunosuppressive medication regimen has been identified as a vital behavior to prevent graft rejection after organ transplantation.2,3 De Geest and colleagues 4 found that medication nonadherence of only $3 \%$ resulted in an increased number of late acute rejections in heart transplant patients. Results of a recent study ${ }^{5}$ in kidney transplant recipients also indicate that medication nonadherence is associated with poor outcomes. Immunosuppressive nonadherence may lead to a number of complications, including acute and chronic rejection, diminished renal function requiring a return to dialysis, or death. ${ }^{6-10}$ Patients returning to dialysis after a failed kidney transplant are at $78 \%$ greater mortality risk than are patients receiving dialysis who are waiting for a transplant. ${ }^{11}$

Much research has been conducted to gain an understanding of medication nonadherence in adult kidney transplant recipients. Research has focused on predictors, ${ }^{6}$ barriers, ${ }^{12}$ and beliefs ${ }^{13}$ of individuals who fail to adhere to immunosuppressive medications. Interventions have also been tested to improve immunosuppressive adherence in this group. ${ }^{14-17}$ No one, however, has analyzed what behaviors or factors are present in successful kidney transplant

Corresponding author: Cynthia L. Russell, RN, PhD, Sinclair School of Nursing, University of Missouri, Columbia, MO 65211 (RussellC@missouri.edu).

To purchase electronic or print reprints, contact: The InnoVision Group, 101 Columbia, Aliso Viejo, CA 92656, Phone (800) 809-2273 (ext 532) or (949) 448-7370 (ext 532), Fax (949) 362-2049, reprints@ aacn.org 
recipients that may contribute to desirable medication adherence and long-term success after kidney transplant. The purpose of this study was to explore the medication-taking behavior of successful kidney transplant recipients and determine what adherence-influencing behaviors were common among this unique group.

\section{Methods}

This study used a qualitative design to explore transplant patients' descriptions of their immunosuppressive medication behaviors. Ethical approval was obtained from the University of Missouri Health Sciences Institutional Review Board. Participants were recruited through an e-mail message sent out to all members of a support group for kidney transplant recipients that includes as its members only recipients who have had their transplant for 25 years or longer. Interested persons could then contact the principal investigator about study participation. All interviews were conducted by telephone, and verbal consent was obtained before each interview. Nineteen kidney transplant recipients were interviewed for this study. Participants were geographically diverse, from various parts of the United States and 1 foreign country. Data were collected through 1-on-1 open-ended telephone interviews and then coded by using a grounded theory approach to explore patterns of phenomena, common descriptions, and themes.

A doctorally prepared nurse researcher (C.R.) with extensive clinical experience in kidney transplant interviewed all participants. Interviews were conducted over the telephone, audio recorded, and professionally transcribed. The nurse researcher asked each subject to discuss why he or she thought that the kidney lasted for such a long time. Probe questions were used to obtain richer descriptions. The length of each interview was about 1 hour. Each interview transcript was individually coded for themes by using the Ethnograph software program (Qualis Research, Colorado Springs, Colorado). The unit of analysis was the complete thought, usually several sentences long. Words and themes were extracted and a code book was created. Using a constant comparative method, the researchers collaboratively reanalyzed and summarized for underlying concepts and themes by using standard qualitative methods. ${ }^{18} \mathrm{~A}$ structure of themes and subthemes was developed through review and comparison of interview transcripts. Revisions were made to the coding categories until all data were coded into mutually exclusive categories.

\section{Results}

The 19 participants ( 7 male, 12 female) interviewed were from 43 to 67 years old, with a mean age of 52.8 years (SD, 6.82). To preserve confidentiality of study participants in this small and exclusive group, we collected a limited amount of demographic data. Participants' transplants were performed between 26 and 36 years ago, with a mean of 30.7 years (SD, 3.2). Thirteen of the 19 participants received kidneys from living donors. Participants were located mainly in the central and northeastern United States. The number of immunosuppressive medications taken was consistent at 1 or 2 daily medications, but the total number of daily medications per participant ranged from 1 to 16 .

After the coding structure was analyzed, 4 general themes related to medication adherence emerged (see Figure): reminder methods, obtaining medications, maintaining routines, and problem-solving strategies. Participants did discuss other factors that they considered important to the longevity of their successful kidney transplants. Those results are being presented in a separate article. In this article, we focus on recipients' medication behavior, as it was a significant part of the interview responses. 


\section{Reminder Methods}

Participants reported that forgetting medications may have been a problem many years ago, but over time they had fewer problems remembering to take medications. Many participants discussed the use of various types of cues to help ensure that their medication is taken regularly. For some, the cues were as simple as keeping the medications in a conspicuous location. Others stored medicines with other items that were associated with habitual or ritualistic behavior. This was particularly useful for participants when traveling. For example:

Well, my little traveling container goes with me ... and actually I'll be going out of town this weekend, so that's coming with me, and it goes in, just my bag that's in by the bathroom, so as you're brushing your teeth, it's like, oh yeah, gotta get my medicine, because typically I'm brushing my teeth after breakfast.

One participant discussed setting a cell phone alarm as a reminder for a medication administration time that did not correspond to another regular event (such as a mealtime or bedtime).

Organization-Several participants described how they have developed systems to organize aspects of their medication-taking behavior. Such systems include tasks such as keeping track of when refills are due, organizing their pills for each week, or always having medication in their pocket or purse in case they away from home at medication-taking time.

Use of a pillbox was mixed, with 8 participants using pillboxes regularly, although 1 of the 8 used the pillbox only for medications other than immunosuppressive agents. Two other participants used pillboxes only when traveling. Participants who did not take their medications as part of a rigid daily routine noted that pillboxes were useful. One participant who not using a pillbox summed up the sentiment the best:

I don't put them in a little Monday to Friday thing, which I probably should, now that I am retired and don't have a real routine in the morning. Because if I forget to take them I don't remember if I did or didn't. You know, it is like such a routine that I go to the sink and take them, then take a shower and if I don't do that routine that morning sometimes I think "did I take them or did I not?" So, I think now I am going to have to start putting them in a different container because I don't know if I did or didn't.

Location-Regardless of pillbox use, most participants kept their medications in a specific location to help remind them to take their medications. One participant emphasized the importance of having one particular spot:

Usually what it is, is my medication is in one spot wherever I live, you know what I am saying? Until I establish that spot, that is when it gets confusing because I know right now I take my pills twice a day, and once I finish my breakfast, I go right to that spot and take my pills. I take the other ones before I go to bed and I always have a glass of milk before I go to bed, so that is the trigger.

For most participants, that spot was in their kitchens, typically out on the counter where the medications themselves become a visual reminder for the participant.

\section{Obtaining Medications}

None of the participants reported much difficulty in being able to obtain their medications, but the ability to obtain medications and the process by which medicines were obtained and costs paid emerged as a theme. One notable characteristic of this group of transplant 
recipients is that they had few problems with insurance coverage. Most participants had insurance coverage with a prescription drug plan requiring monthly copayments of $\$ 20$ per medication per month or less. Some participants paid for their medications out-of-pocket, but they were usually taking generic immunosuppressive agents that had a reasonable cost. All reported having some form of insurance coverage or reimbursement for immunosuppressive medications when they initially received their transplant. Most participants also realized that taking older immunosuppressive agents such as azathioprine and prednisone, which are now available generically, is a benefit to them. As one participant stated when she was discussing what her azathioprine and prednisone costs her,

Yeah, that is cheap, I don't even remember [the cost of Prednisone] and that is the other reason I like the [azathioprine], I don't know what I would do if I were on any of the new [immunosuppressive medicines] without health insurance.

Six participants reported receiving medications by mail, usually 90 days' worth at a time. Some refilled their prescriptions for immunosuppressive medications monthly. Most reported doing so as a matter of personal preference, although a few participants received a financial incentive from their insurance provider to refill for 90 days at time. As one participant described,

Pick up the phone or Internet and order it up. It makes life so much nicer. And the cost-if I went to my local pharmacy and filled a 30-day supply, I would probably pay more than what I pay for a 90-day supply, mail order. You know, it's just how the plans are made up.

Other participants found that using local pharmacies was more convenient for their system of medication management:

I get them through [my pharmacy]. Main reason is that, because I'm so active, and on the go, that I can walk into any [pharmacy], and if I need my medications, they can go ahead and give me a small amount, like if I've forgotten them. There's actually been a couple of trips that I went on, and I walked into the [pharmacy] and I said "I need this, this, and this," and they gave me a small amount.

\section{Forming and Maintaining Routines}

The participants in this study had been taking medication for many years, and all referred to their medication-taking behavior as a habit, for example, "It is just a habit, it's like my habits, because of transplant, are medication, walking, healthy eating."

Many participants described taking medication as just being a part of their daily routine. Several stated that they could not even remember a time when they did not regularly take medication. Several participants did describe, however, that the routine was one of the essential components of successful medication adherence for them. When their routine was broken, it could create difficulties. One participant described what would contribute to the rare occasions when she would for get to take her immunosuppressive medication:

I was out of routine. Something would happen that would cause me to be out of my routine. Like maybe I would be going out to eat breakfast with my mom, and so instead of making breakfast and sitting down and taking my pills I would go out with mom to eat breakfast, and so the pills might not get out. That would be, you know, and I usually hit it even when I do stuff like that, I will put the pills in my pocket and take my pills, but that would be the only time. When I forget, it's because the routine is broken and I didn't think of it.

Formation of the habit of taking medication encompassed not only when but also how the medications were taken. Several participants explained that they developed habits to ensure 
that they took medications with food as necessary, even if the medication time did not coincide with a meal. The participants viewed habit formation as a key to successful medication adherence. They had difficulty, however, explaining what they did to develop the habits.

\section{Problem-Solving Strategies}

Finally, the participants tended to report having developed a set of strategies to overcome potential obstacles to medication adherence. Several described these skills as having formed through trial and error over the years and having become part of their "system" for managing and taking their medications. For example, one participant developed a backup plan for days when she forgot to take her morning immunosuppressant dose:

I would, in a little plastic bag in my desk drawer, just keep my morning medicinedosage of whatever I took in the morning. So, if I got to work and I said "I didn't take my medicine this morning," it would be right there....I always had a backup.

Other participants echoed this idea. Many reported that such medication skills training started early, in the days immediately following transplant. For 1 participant, this created such a strong sense of responsibility for medication adherence that it caused issues later in life:

The key ... is they train you right away to self-medicate, so nurses at [my transplant hospital] when you are recovering from your transplant they didn't bring you pills, you had your own pills and you learned right away that you were in control of your body and how important it was. It gave you a kind of feeling of you are not waiting on anybody else.... And I had struggles with that later on, maybe a year and a half later when I went to the hospital in [city] and they wouldn't hear of me having my own medications, so I had to fight that along the way because many hospitals will not let you have your own medication when you go in.

Participants were asked if they have greater trouble remembering medications when traveling away from home. For those participants who do travel, they described problemsolving strategies they use to remain adherent when their usual routines and reminder cues are disrupted. The various strategies could be classified as using additional cues to prevent missed doses, and taking measures to cope with accidental loss or destruction of medication. As one participant put it, "Well, my little traveling container goes with me, and I always take extra, because you know, you hope that you won't be stranded, but you never know."

Other participants also recounted how, when traveling, they take whatever information they feel they may need to have to get more medication if needed. One participant told us:

And when I travel, I'm always a little bit nervous about something happening to my medication, but usually I just go to [city] or somewhere in the United States, and I always take my little piece of paper with my transplant doctor's name and phone number, in case I have to call him.

This idea was echoed by another participant who had had a situation when traveling in the first few years following her transplant, where she learned the importance of carrying information about her prescriptions when away from home:

I think I did, when I was younger, remember going on a trip to [state], and forgetting all my medication, and having a really hard time, this was like probably a couple of years in. I might have been like 20 or something, and I had a really hard time getting someone to give me the medication, but they did give me the medication in [state], 'cause I explained everything to them, and I think we drove down with my family or something to [location], but otherwise, you know, once I 
explained to them, they knew I needed the medication, and they did give it to me. I don't know if they had to talk to my doctor or whatever...

Such early experiences helped the participants to anticipate potential medication-taking problems and develop strategies to prevent such problems or to handle problems when they arose.

\section{Discussion}

This study is the first to explore the medication-taking behavior of successful kidney transplant recipients who have had their kidney for 25 years or more and to determine what behaviors are common among this group. Four key themes emerged from the data: reminder methods, obtaining medications, maintaining routines, and problem-solving strategies. Participants reported having little difficulty with forgetting to take medications. Through years of experience taking and managing their medications, the participants developed skills and systems that allowed them to be successful in what, for them, was perceived as a very important health behavior for keeping the kidney transplant.

Our findings show that successful medication-taking behavior is dependent not only on knowledge of medication regimens and the medications' necessity, but also on the ability to successfully and conveniently obtain medications. Participants found it important to have a method for obtaining their medications that they perceived as convenient and effective.

This group of successful kidney transplant recipients often described how they associated taking medication with established routines. This finding is congruent with our previous qualitative study ${ }^{13}$ in a group of older and younger adult kidney transplant recipients. Incorporating health behaviors, such taking medication, into established routines may cue the desired behavior without the prerequisite of motivation or remembering. ${ }^{13,19}$ Influencing personal systems and environment through habit formation shows promise for initiating and maintaining behaviors. ${ }^{19}$ Handling disruptions to established routines, whether the disruptions were due to travel, illness, or another factor, has also been cited by our participants and others as a strategy for success. ${ }^{13}$

This group of successful transplant recipients described problem-solving abilities developed over time. Through experience, participants described specific actions taken to ward off anticipated problems with immunosuppressive medication taking. Similar strategies related to timing, dosing, ordering, and filling/using a pillbox have been described. ${ }^{13}$

The depth of understanding provided by this study can assist transplant health care providers in guiding recipients in developing strategies for taking medication similar to those used by successful kidney transplant recipients. Providers can discuss specific actions such as placing medications in conspicuous locations, associating medication taking with routines, and keeping extra medications on hand. Discussing these strategies proactively, to avoid missed or late medications, should be the goal of transplant health care providers.

\section{Limitations}

This study included a racially and ethnically homogenous sample of individuals who received their first transplant more than 25 years ago. Changes in transplantation procedures since that time mean that these recipients' experiences before and after transplant may be quite different from the experiences of current recipients. Additionally, the study participants were predominantly taking "older" immunosuppressive medications such as azathioprine, which have different pharmacokinetics, side effect profiles, and lower costs than many antirejection immunosuppressive agents commonly prescribed today. Although 
the experiences of the participants in this study are a good source of behavioral approaches to effective medication adherence, conceptual models of medication adherence and design of interventions to improve adherence should consider the differences inherent in newer immunosuppressive medications. Future research may also incorporate theoretical sampling approaches that increase sample diversity to develop the themes further, leading to a more generalizable conceptual model of adherence behavior.

\section{Conclusions}

Kidney transplant recipients are dependent on immunosuppressive medications to prevent graft loss and rejection of their kidney. ${ }^{6-10}$ Transplant teams must understand the factors that influence successful medication-taking behavior in this population in order to be able to develop effective interventions to encourage and support positive transplant outcomes.

Interventions that help transplant patients develop medication skills and the ability to establish reminder cues, adjust life routines, and adapt to expected and unexpected disruptions to medication routines may improve medication adherence outcomes and reduce the risk of organ rejection. Additional research is needed to explore how to integrate systemlevel factors that can help address the effect of medication cost on immunosuppressant adherence.

\section{Acknowledgments}

The authors gratefully acknowledge funding support from the John A. Hartford—Atlantic Building Academic Geriatric Nursing Capacity program.

\section{References}

1. Sabate, E. Adherence to Long-Term Therapies: Evidence for Action. Geneva, Switzerland: World Health Organization; 2003.

2. De Geest, S.; Abraham, I.; Dunbar-Jacob, J.; Vanhaecke, J. Behavioral strategies for long-term survival of transplant recipients. In: Metry, J.; Meyer, U., editors. Drug Regimen Compliance: Issues in Clinical Trials and Patient Management. Chichester, NY: John Wiley \& Sons; 1999. p. 163-180.

3. Desmyttere A, Dobbels F, Cleemput I, De Geest S. Noncompliance with immunosuppressive regimen in organ transplantation: is it worth worrying about? Acta Gastroenterol Belgica. 2005; 68(3):347-352.

4. De Geest S, Abraham I, Moons P, et al. Late acute rejection and subclinical noncompliance with cyclosporine therapy in heart transplant recipients. J Heart Lung Transplant. 1998; 17(9):854-863. [PubMed: 9773856]

5. Takemoto SK, Pinsky BW, Schnitzler MA, et al. A retrospective analysis of immunosuppression compliance, dose reduction and discontinuation in kidney transplant recipients. Am J Transplant. 2007; 7(12):2704-2711. [PubMed: 17868065]

6. De Geest S, Borgermans L, Gemoets $\mathrm{H}$, et al. determinants, and consequences of subclinical noncompliance with immunosuppressive therapy in renal transplant recipients. Transplantation. 1995; 59(3):340-347. [PubMed: 7871562]

7. Rosamond W, Flegal K, Furie K, et al. Heart disease and stroke statistics-2008 update: a report from the American Heart Association Statistics Committee and Stroke Statistics Subcommittee. Circulation. 2008; 117(4):125-146.

8. Nevins TE, Kruse L, Skeans MA, Thomas W. The natural history of azathioprine compliance after renal transplantation. Kidney Int. 2001; 60(4):1565-1570. [PubMed: 11576374]

9. Shoskes DA, Avelino L, Barba L, Sender M. Patient death or renal graft loss within 3 yr of transplantation in a county hospital: importance of poor initial graft function. Clin Transplant. 1997; 11(6):618-622. [PubMed: 9408696] 
10. Hawkins DW, Fiedler FP, Douglas HL, Eschbach RC. Evaluation of a clinical pharmacist in caring for hypertensive and diabetic patients. Am J Hospital Pharm. 1979; 36(10):1321-1325.

11. Rao PS, Schaubel DE, Jia X, Li S, Port FK, Saran R. Survival on dialysis post-kidney transplant failure: results from the scientific registry of transplant recipients. Am J Kidney Dis. 2007; 49(2): 294-300. [PubMed: 17261432]

12. Chisholm MA. Identification of medication-adherence barriers and strategies to increase adherence in recipients of renal transplants. Managed Care Interface. 2004; 17(9):44-48. [PubMed: 15521285]

13. Russell CL, Kilburn E, Conn VS, Libbus MK, Ashbaugh C. Medication taking beliefs of adult renal transplant recipients. Clin Nurse Specialist. 2003; 17(4):200-208.

14. Chisholm MA, Mulloy LL, Jagadeesan M, DiPiro JT. Impact of clinical pharmacy services on renal transplant patients' compliance with immunosuppressive medications. Clin Transplant. 2001; 15(5):330-336. [PubMed: 11678959]

15. Hardstaff R, Green K, Talbot D. Measurement of compliance posttransplantation-the results of a 12-month study using electronic monitoring. Transplant Proc. 2003; 35(2):796-797. [PubMed: 12644142]

16. De Geest S, Schafer-Keller P, Denhaerynck K, et al. Supporting medication adherence in renal transplantation (SMART): a pilot RCT to improve adherence to immunosuppressive regimens. Clin Transplant. 2006; 20(3):359-368. [PubMed: 16824155]

17. Chisholm MA, Vollenweider LJ, Mulloy LL, et al. Renal transplant patient compliance with free immunosuppressive medications. Transplantation. 2000; 70(8):1240-1244. [PubMed: 11063348]

18. Corbin, J.; Strauss, A. Basics of Qualitative Research: Techniques and Procedures for Developing Grounded Theory. 3. Thousand Oaks, CA: Sage Publications; 2008.

19. Alemi F, Pawloski L, Fallon WF Jr. System thinking in a personal context to improve eating behaviors. J Healthcare Qual. 2003; 25(2):20-25. 


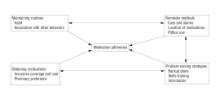

Figure.

Four general themes related to medication adherence. 